\title{
ROTUNDITY IN LEBESGUE-BOCHNER FUNCTION SPACES
} BY

\author{
MARK A. SMITH AND BARRY TURETT
}

\begin{abstract}
This paper concerns the isometric theory of the LebesgueBochner function space $L^{P}(\mu, X)$ where $1<p<\infty$. Specifically, the question of whether a geometrical property lifts from $X$ to $L^{P}(\mu, X)$ is examined. Positive results are obtained for the properties local uniform rotundity, weak uniform rotundity, uniform rotundity in each direction, midpoint local uniform rotundity, and $B$-convexity. However, it is shown that the RadonRiesz property does not lift from $X$ to $L^{P}(\mu, X)$. Consequently, LebesgueBochner function spaces with the Radon-Riesz property are examined more closely.
\end{abstract}

1. Introduction. Frequently in the literature authors have proved theorems stating that the Banach sequence space $l^{P}(X)$ has a certain geometric property exactly when the Banach spaces $X$ and $l^{p}$ have that same property [1], [4], [5], [11], [13], [18], [25]. Less frequently, however, do authors consider the corresponding question for the Lebesgue-Bochner function space $L^{p}(\mu, X)$ (see [3] for a discussion of this space). Although most people are willing to believe an affirmative answer to such a question, the proof of such a response is often nontrivial. In 1941, Day [4] proved that $L^{p}(\mu, X)$ for $1<p<\infty$ is uniformly rotund whenever $X$ is uniformly rotund by defining an embedding from the simple functions of $L^{p}(\mu, X)$ into the space $l^{p}(X)$ and then applying the corresponding result that he proved for $l^{p}(X)$. Subsequently, McShane [14] gave a direct proof of Day's result. It is well known [5] and much more easily proved that an analogous theorem holds if uniform rotundity is replaced by rotundity (strict convexity).

In this paper, theorems of the same ilk are obtained for several convexity properties that lie in strength between uniform rotundity (UR) and rotundity (R) as well as for some other geometrical notions. In particular, if $X$ is locally uniformly rotund (LUR) and $1<p<\infty$, it is shown that $L^{p}(\mu, X)$ is also locally uniformly rotund. This answers a question posed to us by $\mathrm{J}$. Diestel. Similar theorems are obtained for $X$ weakly uniformly rotund (WUR), uniformly rotund in every direction (URED) or midpoint locally uniformly rotund (MLUR). To avoid confusion, the relative strengths of these distinct rotundity notions are given in the chart below where an arrow denotes implication.

Presented to the Society, January 4, 1978; received by the editors January 23, 1978.

AMS (MOS) subject classifications (1970). Primary 46B99, 46E40.

Key words and phrases. Lebesgue-Bochner function space, locally uniformly rotund, weakly uniformly rotund, $B$-convex, uniformly non- $l^{1}(n)$, Radon-Riesz property. 


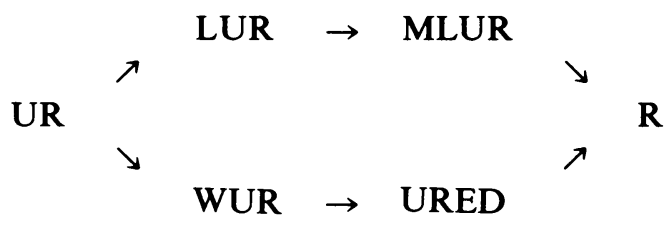

All of these properties are known to carry over from $X$ to $l^{p}(X)$ for $1<p<\infty$. However, neither Day's embedding argument nor McShane's proof for the uniform rotundity case seems adaptable to prove the theorems concerning these generalizations of uniform rotundity in $L^{p}(\mu, X)$. Here, a technique is presented that with the appropriate modification of details yields the proofs of all four of the theorems indicated above. But it should be noted that this technique does not seem adaptable to prove Day's result.

In contrast to these positive results, an example is given of a reflexive Banach space $X$ with the Radon-Riesz property, called property $(H)$ in Day [6, p. 146], for which $L^{p}([0,1], \lambda, X)$ fails to have the Radon-Riesz property for each $1 \leqslant p<\infty$. This answers a question posed by $\mathrm{E}$. Leonard [11, $\mathrm{p}$. 256]. This result deviates from the pattern set above since it is known that if $X$ has the Radon-Riesz property, then $l^{p}(X)$ for $1 \leqslant p<\infty$ also has the Radon-Riesz property [1], [11]. Consequently, Lebesgue-Bochner function spaces having the Radon-Riesz property are examined more closely.

Finally, it is shown that if $X$ is uniformly non- $l^{1}(n)$, then $l^{p}(X)$ and $L^{P}(\mu, X)$ are also uniformly non- $l^{1}(n)$. This result generalizes the known theorem, due to T. Figiel [17], that $l^{p}(X)$ and $L^{p}(\mu, X)$ are $B$-convex whenever $X$ is $B$-convex.

It should be noted that if $L^{p}(\mu, X)$ has a property inherited by subspaces, then both $X$ and $L^{p}(\mu)$, each being isometrically isomorphic to a subspace of $L^{p}(\mu, X)$, necessarily have that property. Since $L^{p}(\mu)$ for $1<p<\infty$ is uniformly rotund, it has all the geometrical properties examined in this paper and so the problems considered here are ones of lifting a geometrical property from $X$ to $L^{p}(\mu, X)$.

The authors wish to thank Professors L. Dor, T. Morley and J. J. Uhl, Jr. for some helpful comments.

2. A Banach space $X$ is said to be locally uniformly rotund [13] if, for each $\varepsilon>0$ and each $x \in X$ with $\|x\|=1$, there exists $\delta(x, \varepsilon)>0$ such that if $y \in X$ with $\|y\|=1$ and $\|x-y\| \geqslant \varepsilon$, then $\left\|\frac{1}{2}(x+y)\right\|<1-\delta(x, \varepsilon)$. In order to characterize locally uniformly rotund Lebesgue-Bochner function spaces, an equivalent form of this definition is needed.

LEMMA 1. A Banach space $X$ is locally uniformly rotund if and only if, for each $\varepsilon>0$ and each $x \in X$ with $\|x\|=1$, there exists $\delta^{\prime}(x, \varepsilon)>0$ such that if $\|y\|<1+2 \delta^{\prime}(x, \varepsilon)$ and $\|x-y\|>\varepsilon$, then $\left\|\frac{1}{2}(x+y)\right\|<1-\delta^{\prime}(x, \varepsilon)$.

Proof. Assume $X$ is locally uniformly rotund, and let $\varepsilon>0$ and $x \in X$ with $\|x\|=1$ be given. Let $\delta(x, \varepsilon / 2)$ be the number guaranteed in the definition of a locally uniformly rotund space and set $\delta^{\prime}(x, \varepsilon)=\frac{1}{2} \delta(x, \varepsilon / 2)$. 
If $y \in X$ and $\|y\|<1-2 \delta^{\prime}(x, \varepsilon)$, then $\|x+y\|<\|x\|+\|y\|<$ $2\left(1-\delta^{\prime}(x, \varepsilon)\right)$.

So consider the case $|1-\|y\|| \leqslant 2 \delta^{\prime}(x, \varepsilon)$ and $\|x-y\| \geqslant \varepsilon$. Let $z=$ $y /\|y\|$. Then $\|z-y\|=|1-\|y\|| \leqslant \delta(x, \varepsilon / 2)$. Also $\|x-z\| \geqslant \varepsilon / 2$. (Indeed, if $\|x-z\|<\varepsilon / 2$, then $\varepsilon \leqslant\|x-y\|<(\varepsilon / 2)+\delta(x, \varepsilon / 2) \leqslant 3 \varepsilon / 4$ since $\delta(x, \varepsilon / 2)<\varepsilon / 4$.) Hence, since $X$ is locally uniformly rotund, $\|x+y\|<\| x$ $+z\|+\| y-z \| \leqslant 2(1-\delta(x, \varepsilon / 2))+\delta(x, \varepsilon / 2)=2\left(1-\delta^{\prime}(x, \varepsilon)\right)$.

Since the sufficiency is clear, the proof of the lemma is complete.

The first theorem characterizes locally uniformly rotund Lebesgue-Bochner function spaces over finite measure spaces. Its proof will serve as a model for later proofs.

TheOREM 2. Let $(S, \Sigma, \mu)$ be a finite measure space. If $X$ is a locally uniformly rotund Banach space and $1<p<\infty$, then $L^{p}(\mu, X)$ is locally uniformly rotund.

Proof. For convenience, assume $\mu(S)=1$. The proof for general finite measure spaces requires only minor modifications.

Let $\varepsilon>0$ be given and suppose $f$ is a norm-one function in $L^{p}(\mu, X)$. Since $\|f(\cdot)\|^{p} \in L^{1}(\mu)$ there exists $\alpha$ with $0<\alpha<1$ such that if $A \in \Sigma$ with $\mu(A)<\alpha$, then $\left\|f \chi_{A}\right\|_{p} \leqslant \varepsilon / 16$. Choose $M>1$ such that the set $F \equiv\{s \in S$ : $M^{-1}<\|f(s)\|<M$ \} has positive measure and $\mu$ (support $\left.f \backslash F\right)<\alpha / 2$. Now choose $m>M$ such that $1 / m \leqslant \varepsilon / 16$ and the set $E \equiv\{s \in F$ : $\left.\delta^{\prime}(f(s) /\|f(s)\|, \varepsilon / 4 M) \geqslant M / m\right\}$, where $\delta^{\prime}$ is given in Lemma 1, has positive measure and $\mu$ (support $f \backslash E)<\alpha$. Set $\eta=(\alpha \mu(F))^{1 / p} / m$ and note $0<\eta<$ $1 / m$.

Let $g$ in $L^{p}(\mu, X)$ satisfy $\|g\|_{p}=1$ and $\|f-g\|_{p}>\varepsilon$.

If $\|(\|f(\cdot)\|-\|g(\cdot)\|)\|_{p} \geqslant \eta$, then since $L^{p}(\mu)$ is uniformly rotund there exists $\delta(\eta)>0$ such that $\|f+g\|_{p} \leqslant\|(\|f(\cdot)\|+\|g(\cdot)\|)\|_{p} \leqslant 2(1-\delta(\eta))$.

Now consider the case $\|(\|f(\cdot)\|-\|g(\cdot)\|)\|_{p}<\eta$. Let $G=\{s \in E: \mid(\|f(s)\|$ $-\|g(s)\|) \mid<1 / m\}$. Note $\mu(E \backslash G)<\alpha$ since otherwise, by the definition of $G$,

$$
\begin{aligned}
\|(\|f(\cdot)\|-\|g(\cdot)\|)\|_{p} & >\left\|(\|f(\cdot)\|-\|g(\cdot)\|) \chi_{E \backslash G}\right\|_{p} \\
& >\mu(E \backslash G)^{1 / p} / m>\alpha^{1 / p} / m>\eta,
\end{aligned}
$$

a contradiction. From this and since $\mu$ (support $f \backslash E)<\alpha$,

$$
\left\|f \chi_{E \backslash G}\right\|_{p} \leqslant \varepsilon / 16 \text { and }\left\|f \chi_{S \backslash E}\right\|_{p} \leqslant \varepsilon / 16 \text {. }
$$

If $\left\|g \chi_{S \backslash E}\right\|_{p}>\varepsilon / 4$, then, by (1), $\left\|(\|g(\cdot)\|-\|f(\cdot)\|) \chi_{S \backslash E}\right\|_{p}>\left\|g \chi_{S \backslash E}\right\|_{p}-$ $\left\|f \chi_{S \backslash E}\right\|_{p}>3 \varepsilon / 16>\eta$, again a contradiction. Therefore $\left\|g \chi_{S \backslash E}\right\|_{p}<\varepsilon / 4$ and hence by (1)

$$
\left\|(f-g) \chi_{S \backslash E}\right\|_{p}<5 \varepsilon / 16
$$


Now if $\left\|(f-g) \chi_{S \backslash G}\right\|_{p}>\varepsilon / 2$, then, from (2) and the triangle inequality,

$$
\begin{aligned}
\left\|f \chi_{E \backslash G}\right\|_{P}+\left\|g \chi_{E \backslash G}\right\|_{P} & >\left\|(f-g) \chi_{E \backslash G}\right\|_{p} \\
& =\left\|(f-g) \chi_{S \backslash G}-(f-g) \chi_{S \backslash E}\right\|_{P}>3 \varepsilon / 16
\end{aligned}
$$

and hence, by (1), $\left\|g \chi_{E \backslash G}\right\|_{p}>\varepsilon / 8$. This implies $\left\|(\|g(\cdot)\|-\|f(\cdot)\|) \chi_{E \backslash G}\right\|_{p}$ $>\left\|g \chi_{E \backslash G}\right\|_{p}-\left\|f \chi_{E \backslash G}\right\|_{p}>\varepsilon / 16>\eta$, a contradiction. Therefore $\|(f-$ g) $\chi_{S \backslash G} \|_{p}<\varepsilon / 2$ and so

$$
\left\|(f-g) \chi_{G}\right\|_{p}>\varepsilon / 2 .
$$

Let $B=\{s \in G:\|f(s)-g(s)\|>\varepsilon / 4\}$. Then $\mu(B)>\alpha$ since otherwise, by (3) and Minkowski's inequality,

$$
\begin{aligned}
\varepsilon / 2< & \left\|(f-g) \chi_{G}\right\|_{p} \\
< & \left.<\int_{B}\|f(s)-g(s)\|^{p} d \mu(s)+\int_{G \backslash B}\|f(s)-g(s)\|^{p} d \mu(s)\right]^{1 / p} \\
< & {\left[\int_{B}\left(\|f(s)\|+\|g(s)\|^{p} d \mu(s)+\int_{G \backslash B}\left(\frac{\varepsilon}{4}\right)^{p} d \mu(s)\right]^{1 / p}\right.} \\
& <\left[\int_{B}\left(2\|f(s)\|+\frac{1}{m}\right)^{p} d \mu(s)+\int_{G \backslash B}\left(\frac{\varepsilon}{4}\right)^{p} d \mu(s)\right]^{1 / p} \\
& <\left[\int_{B}\left(2\|f(s)\|+\frac{1}{m}\right)^{p} d \mu(s)\right]^{1 / p}+\left[\int_{G \backslash B}\left(\frac{\varepsilon}{4}\right)^{p} d \mu(s)\right]^{1 / p} \\
& <2\left\|f \chi_{B}\right\|_{p}+\mu(B)^{1 / p} / m+\varepsilon \mu(G \backslash B)^{1 / p} / 4 \\
& <\varepsilon / 8+\varepsilon / 16+\varepsilon / 4,
\end{aligned}
$$

a contradiction.

Now, from the definitions of $B, G$ and $E$, for all $s \in B$,

$$
\left\|\frac{f(s)}{\|f(s)\|}-\frac{g(s)}{\|f(s)\|}\right\|>\frac{\varepsilon}{4}\|f(s)\|>\frac{\varepsilon}{4 M}
$$

and

$$
\begin{aligned}
\left\|\frac{g(s)}{\|f(s)\|}\right\| & <\frac{\|f(s)\|+1 / m}{\|f(s)\|} \\
& <1+\frac{M}{m}<1+\delta^{\prime}\left(\frac{f(s)}{\|f(s)\|}, \frac{\varepsilon}{4 M}\right) .
\end{aligned}
$$

Therefore by Lemma 1 for $s \in B$

$$
\|f(s)+g(s)\|<2(1-M / m)\|f(s)\| .
$$

Also, for $s \in B \subset G,\|f(s)\|-\|g(s)\|<1 / m<M\|f(s)\| / m$ and hence

$$
(1-M / m)\|f(s)\|<\|g(s)\| .
$$


From (4), (5) and Minkowski's inequality,

$$
\begin{aligned}
\|f+g\|_{p}< & {\left[\int_{S \backslash B}(\|f(s)\|+\|g(s)\|)^{p} d \mu(s)\right.} \\
& \left.+\int_{B}\left(2\left(1-\frac{M}{m}\right)\|f(s)\|\right)^{p} d \mu(s)\right]^{1 / p} \\
< & {\left[\int_{S \backslash B}\|f(s)\|^{p} d \mu(s)+\int_{B}\left(1-\frac{M}{m}\right)^{p}\|f(s)\|^{p} d \mu(s)\right]^{1 / p} } \\
& +\left[\int_{S \backslash B}\|g(s)\|^{p} d \mu(s)+\int_{B}\left(1-\frac{M}{m}\right)^{p}\|f(s)\|^{p} d \mu(s)\right]^{1 / p} \\
< & {\left[\|f\|_{p}^{p}-\int_{B}\left(1-\left(1-\frac{M}{m}\right)^{p}\right)\|f(s)\|^{p} d \mu(s)\right]^{1 / p}+\|g\|_{p} } \\
< & {\left[1-\left(1-(1-M / m)^{p}\right) \alpha / M^{p}\right]^{1 / p}+1 . }
\end{aligned}
$$

Note that $1-(1-M / m)^{p}>0$ since $m>M$.

Thus, in either case, the norm of the midpoint of $f$ and $g$ is bounded by a number less than one and independent of $g$. This completes the proof of Theorem 2.

A different sort of generalization of uniform rotundity is weak uniform rotundity. A Banach space $X$ is said to be weakly uniformly rotund [20] if, for each $\varepsilon>0$ and each $x^{*} \in X^{*}$ with $\left\|x^{*}\right\|=1$, there exists $\delta\left(x^{*}, \varepsilon\right)>0$ such that if $x$ and $y$ are in $X$ with $\|x\|=\|y\|=1$ and $x^{*}(x-y)>\varepsilon$, then $\left\|\frac{1}{2}(x+y)\right\|<1-\delta\left(x^{*}, \varepsilon\right)$. The next lemma is similar to Lemma 1 and therefore its proof is omitted.

LEMMA 3. A Banach space $X$ is weakly uniformly rotund if and only if, for each norm-one $x^{*} \in X^{*}$ and each $\varepsilon>0$, there exists $\delta^{\prime}\left(x^{*}, \varepsilon\right)>0$ such that if $\|x\|=1,\|y\|<1+2 \delta^{\prime}\left(x^{*}, \varepsilon\right)$ and $x^{*}(x-y)>\varepsilon$, then $\left\|\frac{1}{2}(x+y)\right\|<1-$ $\delta^{\prime}\left(x^{*}, \varepsilon\right)$.

From the definition it is clear that a discussion of weak uniform rotundity in a space is facilitated by some knowledge of its dual space. In order that the dual of the Lebesgue-Bochner space $L^{p}(\mu, X)$ be tractable, in Theorem 4, $X^{*}$ is assumed to have the Radon-Nikodým property with respect to $\mu$. It is well known (see [7, IV. 1.1]) that for $(S, \Sigma, \mu)$ a finite measure space and $1<p<\infty$, the dual of $L^{p}(\mu, X)$ is $L^{q}\left(\mu, X^{*}\right)$ where $p^{-1}+q^{-1}=1$ if and only if $X^{*}$ has the Radon-Nikodým property with respect to $\mu$.

THEOREM 4. Let $(S, \Sigma, \mu)$ be a finite measure space. If $1<p<\infty$ and $X$ is a Banach space that is weakly uniformly rotund and whose dual space $X^{*}$ has the Radon-Nikodym property with respect to $\mu$, then $L^{p}(\mu, X)$ is weakly uniformly rotund. 
Proof. Again assume $\mu(S)=1$. Let $H \in L^{p}(\mu, X)^{*}=L^{q}\left(\mu, X^{*}\right)$ be of norm one and let $\varepsilon>0$ be given. Since $\|H(\cdot)\|^{q} \in L^{1}(\mu)$ there exists $\alpha$ with $0<\alpha<1$ such that if $\mu(A)<\alpha$ for $A \in \Sigma$, then $\left\|H \chi_{A}\right\|_{q} \leqslant \varepsilon / 16$. Choose $M>1$ such that the set $F \equiv\left\{s \in S: M^{-1} \leqslant\|H(s)\|<M\right\}$ has positive measure and $\mu$ (support $H \backslash F)<\alpha / 2$. Now choose $m>M$ such that the set $E \equiv\left\{s \in F: \delta^{\prime}\left(H(s) /\|H(s)\|, \varepsilon \alpha^{1 / p} / 4^{1+1 / p} M\right)>1 / m\right\}$, where $\delta^{\prime}$ is given in Lemma 3 , has positive measure and $\mu($ support $H \backslash E)<\alpha$. Set $\eta=$ $\varepsilon(\alpha \mu(F))^{1 / p} / 16 M m$.

Let $f$ and $g$ in $L^{p}(\mu, X)$ satisfy $\|f\|_{p}=\|g\|_{p}=1$ and $H(f-g)>\varepsilon$.

As in Theorem 2, if $\|(\|f(\cdot)\|-\|g(\cdot)\|)\|_{p}>\eta$, then the uniform rotundity of $L^{p}(\mu)$ forces the norm of the midpoint of $f$ and $g$ to be less than $1-\delta(\eta)$.

So assume $\|(\|f(\cdot)\|-\|g(\cdot)\|)\|_{p}<\eta$. Define $G=\{s \in E: \mid(\|f(s)\|-$ $\|g(s)\|) \mid<\varepsilon / 16 M m\}$. Note that $\mu(E \backslash G)<\alpha$. Therefore by the choice of $\alpha$

$$
\begin{aligned}
\int_{S \backslash G} H(s)[f(s)-g(s)] d \mu(s) & <\left\|H \chi_{s \backslash G}\right\|_{q}\left\|(f-g) \chi_{s \backslash G}\right\|_{p} \\
& <\left(\left\|H \chi_{s \backslash E}\right\|_{q}+\left\|H \chi_{E \backslash G}\right\|_{q}\right)\left(\|f\|_{p}+\|g\|_{p}\right) \\
& <\varepsilon / 4 .
\end{aligned}
$$

Hence since $H(f-g) \geqslant \varepsilon$

$$
\int_{G} H(s)[f(s)-g(s)] d \mu(s)>\varepsilon / 2 .
$$

Define $P=\{s \in G: H(s)[f(s)-g(s)]>\varepsilon / 4\}$. Then $\mu(P) \geqslant \alpha$ since otherwise by (1)

$$
\begin{aligned}
\frac{\varepsilon}{2} & <\int_{P} H(s)[f(s)-g(s)] d \mu(s)+\int_{G \backslash P} H(s)[f(s)-g(s)] d \mu(s) \\
& <\left\|H \chi_{P}\right\|_{q}\left(\|f\|_{P}+\|g\|_{p}\right)+\varepsilon \mu(G \backslash P) / 4<3 \varepsilon / 8 .
\end{aligned}
$$

Also for each $s \in P$

$$
\|f(s)\| \geqslant \varepsilon / 16 M
$$

since otherwise there exists $s \in P \subset G \subset F$ such that $\varepsilon / 4<H(s)[f(s)-$ $g(s)]<M(2\|f(s)\|+\varepsilon / 16 M m) \leqslant 3 \varepsilon / 16$.

Finally, define $B=\left\{s \in P:\|f(s)\|<(4 / \alpha)^{1 / p}\right\}$. Then $\mu(B)>\alpha / 2$ since otherwise $\mu(P \backslash B) \geqslant \alpha / 2$ and hence $1>\left\|f \chi_{P \backslash B}\right\|_{p}>(4 \mu(P \backslash B) / \alpha)^{1 / p}>$ $2^{1 / p}$.

Now, from (2) and the definitions of $B, P, G, E$, and $F$, for each $s \in B$

$$
\frac{H(s)}{\|H(s)\|}\left[\frac{f(s)}{\|f(s)\|}-\frac{g(s)}{\|f(s)\|}\right]>\frac{\varepsilon \alpha^{1 / p}}{4^{1+1 / p} M}
$$

and

$$
\left\|\frac{g(s)}{\|f(s)\|}\right\|<1+\delta^{\prime}\left(\frac{H(s)}{\|H(s)\|}, \frac{\varepsilon \alpha^{1 / p}}{4^{1+1 / p} M}\right) .
$$

Therefore by Lemma 3 for $s \in B$

$$
\|f(s)+g(s)\|<2(1-1 / m)\|f(s)\| .
$$


Also, for $s \in B \subset G,\|f(s)\|-\|g(s)\|<\|f(s)\| / m$ and hence

$$
(1-1 / m)\|f(s)\|<\|g(s)\| \text {. }
$$

Now, as in the proof of Theorem 2, from (3), (4) and Minkowski's inequality,

$$
\begin{aligned}
\|f+g\|_{p} & <\left[\int_{S \backslash B}(\|f(s)\|+\|g(s)\|)^{p} d \mu(s)\right. \\
& \left.+\int_{B}\left(2\left(1-\frac{1}{m}\right)\|f(s)\|\right)^{p} d \mu(s)\right]^{1 / p} \\
& <\left[1-\left(1-(1-1 / m)^{p}\right)(\varepsilon / 16 M)^{p} \alpha / 2\right]^{1 / p}+1 .
\end{aligned}
$$

This completes the proof of Theorem 4.

A natural concern is that the hypothesis that $X^{*}$ has the Radon-Nikodým property with respect to $\mu$ in Theorem 4 is restrictive. However, there is no known weakly uniformly rotund Banach space whose dual space does not have the Radon-Nikodým property. So perhaps such a concern will be proved to be unwarranted.

A more general notion than weak uniform rotundity is uniformly rotund in every direction, a property that characterizes those Banach spaces in which every bounded set has at most one Čebyšev center. A Banach space $X$ is uniformly rotund in every direction [8] if, for each $\varepsilon>0$ and nonzero $z \in X$, there exists $\delta(z, \varepsilon)>0$ such that if $x$ and $y$ are in $X$ with $\|x\|=\|y\|=1$, $\|x-y\|>\varepsilon$ and $x-y=\alpha z$ for some $\alpha$, then $\left\|\frac{1}{2}(x+y)\right\|<1-\delta(z, \varepsilon)$. A theorem analogous to Theorem 2 is obtained below for this geometrical notion. Since its proof is similar to that of Theorems 2 and 4 some of the computations in the proof are omitted. Again, before proceeding to the theorem an equivalent formulation of this property is needed.

LEMMA 5. A Banach space $X$ is uniformly rotund in every direction if and only if, for each $\varepsilon>0$ and norm-one $z \in X$, there exists $\delta^{\prime}(z, \varepsilon)>0$ such that if $\|x\|=1,\|y\|<1+2 \delta^{\prime}(z, \varepsilon), x-y=\alpha z$ for some $\alpha$, and $\|x-y\|>\varepsilon$, then $\left\|\frac{1}{2}(x+y)\right\|<1-\delta^{\prime}(z, \varepsilon)$.

TheOREM 6. Let $(S, \Sigma, \mu)$ be a finite measure space. If $X$ is a Banach space that is uniformly rotund in every direction and $1<p<\infty$, then $L^{p}(\mu, X)$ is also uniformly rotund in every direction.

Proof. Assume $\mu(S)=1$. Let $\varepsilon>0$ be given and suppose $h$ is a norm-one function in $L^{p}(\mu, X)$. Since $\|h(\cdot)\|^{p} \in L^{1}(\mu)$ there exists $\alpha$ with $0<\alpha<1$ such that if $A \in \Sigma$ with $\mu(A)<\alpha$, then $\left\|h \chi_{1}\right\|_{p}<1 / 8$. Choose $m>1$ such that the set $E \equiv\left\{s \in\right.$ support $\left.h: \delta^{\prime}\left(h(s) /\|h(s)\|, \varepsilon \alpha^{1 / p} / 4^{1+1 / p}\right)>1 / m\right\}$ satisfies $\mu(E)>0$ and $\mu$ (support $h \backslash E)<\alpha$. Set $\eta=\varepsilon \alpha^{1 / p} / 16 m$.

Let $f$ and $g$ be norm-one functions in $L^{P}(\mu, X)$ such that $f-g=\varepsilon h$.

Suppose $\|(\|f(\cdot)\|-\|g(\cdot)\|)\|_{p}<\eta$. (Otherwise the proof is completed as before.) Let $G=\{s \in E$ : $|(\|f(s)\|-\|g(s)\|)|<\varepsilon / 16 m\}$. Note $\mu(E \backslash G)<\alpha$. 
This and the fact that $\mu$ (support $h \backslash E)<\alpha$ imply that $\left\|(f-g) \chi_{s \backslash G}\right\|_{p}<\varepsilon / 4$. Therefore since $\|f-g\|_{p}=\varepsilon$

$$
\left\|(f-g) \chi_{G}\right\|_{p}>\varepsilon / 2 \text {. }
$$

Define $P=\{s \in G:\|h(s)\|>1 / 4\}$. Then $\mu(P)>\alpha$. Also for each $s \in P$

$$
\|f(s)\|>\varepsilon / 16
$$

Now define $B=\left\{s \in P:\|f(s)\|<(4 / \alpha)^{1 / p}\right\}$ and note that $\mu(B)>\alpha / 2$. From (2) and the definitions of $B, P, G$, and $E$, for each $s \in B$

$$
\frac{f(s)}{\|f(s)\|}-\frac{g(s)}{\|f(s)\|}=\frac{\varepsilon}{\|f(s)\|} h(s), \quad\left\|\frac{f(s)}{\|f(s)\|}-\frac{g(s)}{\|f(s)\|}\right\|>\frac{\varepsilon \alpha^{1 / p}}{4^{1+1 / p}},
$$

and

$$
\left\|\frac{g(s)}{\|f(s)\|}\right\|<1+\delta^{\prime}\left(\frac{h(s)}{\|h(s)\|}, \frac{\varepsilon \alpha^{1 / p}}{4^{1+1 / p}}\right) .
$$

Therefore by Lemma 5 for $s \in B$

$$
\|f(s)+g(s)\|<2(1-1 / m)\|f(s)\| .
$$

Also for $s \in B \subset G$

$$
(1-1 / m)\|f(s)\|<\|g(s)\| .
$$

Now, from (2), (3), (4), and Minkowski's inequality,

$$
\|f+g\|_{p}<\left[1-\left(1-(1-1 / m)^{p}\right)(\varepsilon / 16)^{p} \alpha / 2\right]^{1 / p}+1 .
$$

This completes the proof of Theorem 6.

Another generalization of local uniform rotundity is midpoint local uniform rotundity. This property was introduced in [1] and initially studied as a candidate for the rotundity notion dual to the smoothness notion of Fréchet differentiability of the norm. A Banach space $X$ is midpoint locally uniformly rotund if, for each $\varepsilon>0$ and $w \in X$ with $\|w\|=1$, there exists $\delta(w, \varepsilon)>0$ such that if $x$ and $y$ are in $X$ with $\|x\|=\|y\|=1$ and $\|x-y\|>\varepsilon$, then $\|x+y-2 w\|>\delta(w, \varepsilon)$. The course should now be clear. A theorem analogous to the ones above can be obtained by the same technique. For the sake of space, the proof of this theorem and even the statement of the lemma that reformulates this notion into usable form are omitted.

TheOREM 7. Let $(S, \Sigma, \mu)$ be a finite measure space. If $X$ is a Banach space that is midpoint locally uniformly rotund and $1<p<\infty$, then $L^{p}(\mu, X)$ is also midpoint locally uniformly rotund.

The preceding theorems suggest that most rotundity properties carry over from $X$ to $L^{p}(\mu, X), 1<p<\infty$. However, there are geometric properties that hold for a Banach space $X$ but not for $L^{P}(\mu, X)$. The Radon-Riesz property is one such property. A Banach space is said to have the RadonRiesz property if every sequence of norm-one elements that converges weakly to a norm-one element converges in norm (that is, for sequences in the unit sphere norm and weak convergence coincide). Radon [15] and Riesz [16] 
proved that $L^{p}(\mu)$ for $1<p<\infty$ has this property. It is easy to show that every locally uniformly rotund (and hence every uniformly rotund) Banach space has the Radon-Riesz property. Also it is immediate that every Banach space with the Schur property (coincidence of norm and weak convergence of all sequences), for example, $l^{1}$ or any finite dimensional space, has the Radon-Riesz property. So in general the Radon-Riesz property does not imply rotundity.

Although the hoped-for duality for midpoint local uniform rotundity did not eventuate, Anderson [1] did show that in reflexive spaces the combination of rotundity and the Radon-Riesz property implies midpoint local uniform rotundity and is in full duality with Fréchet differentiability of the conjugate norm.

The next thoerem shows that in the context of Lebesgue-Bochner function spaces the Radon-Riesz property is a condition stronger than rotundity and hence this property does not lift from $X$ to $L^{p}(\mu, X)$.

TheOREM 8. Let $(S, \Sigma, \mu)$ be a measure space that is not purely atomic and let $1<p<\infty$. If $L^{p}(\mu, X)$ has the Radon-Riesz property, then $X$ (and hence $\left.L^{p}(\mu, X)\right)$ is rotund.

Proof. If $X$ is not rotund, there exist $x$ and $z$ in $X$ such that $\|x\|=\| x \pm$ $z \|=1$ and $z \neq 0$. Let $T \in \Sigma$ be a set of positive, finite measure that contains no atoms and let $r_{n}$ denote the " $n$th Rademacher function" defined on $T$; $r_{n}(s)=0$ for $s \in S \backslash T$. For ease, assume $\mu(T)=1$. Now define $f_{n}$ and $f$ in $L^{p}(\mu, X)$ by $f_{n}(s)=x \chi_{T}(s)+r_{n}(s) z$ and $f(s)=x \chi_{T}(s)$ for $s \in S$. Note that $f_{n}(s)-f(s)=r_{n}(s) z$. Therefore $\left\|f_{n}\right\|_{p}=\|f\|_{p}=1$ and $\left\|f_{n}-f\right\|_{p}=\|z\|>0$. The proof is completed by noting that $\left(f_{n}\right)$ converges weakly to $f$ in $L^{p}(\mu, X)$ since $\left(r_{n}\right)$ converges weakly to zero in $L^{P}(\mu)$.

An application of Theorem 8 with $X$ the two-dimensional $l^{1}$ space yields the following corollary.

COROLlary 9. There exists a reflexive Banach space $X$ with the Radon-Riesz property such that $L^{p}([0,1], \lambda, X)$ does not have the Radon-Riesz property for any $1<p<\infty$. $(\lambda$ denotes Lebesgue measure on $[0,1]$.)

Another interesting consequence in the setting of this theorem is that for reflexive Lebesgue-Bochner function spaces the Radon-Riesz property is in full duality with Fréchet differentiability of the conjugate norm. This follows from Theorem 8 and the duality mentioned before it. Note the fact that $L^{P}(\mu, X)$ for $1<p<\infty$ is reflexive if and only if $X$ is reflexive follows easily from the statement immediately preceding Theorem 4 .

Since both rotundity and the Radon-Riesz property in $X$ are necessary conditions for $L^{p}(\mu, X)$ to have the Radon-Riesz property, it is natural to ask if they are sufficient.

Question 10. If $X$ is a rotund Banach space with the Radon-Riesz property and $1<p<\infty$, does $L^{p}(\mu, X)$ have the Radon-Riesz property? 
This question has an affirmative answer whenever $X$ is reflexive. This follows from the now familiar duality and a theorem of Leonard and Sundaresan [12] stating that if $1<p<\infty, X$ and $L^{p}(\mu, X)$ have or fail to have Fréchet differentiable norms together. With this as supporting evidence along with the fact that the combination of rotundity and the Radon-Riesz property is a condition lying in strength between local uniform rotundity and rotundity, each of which lifts from $X$ to $L^{p}(\mu, X)$, an affirmative answer to this question might be expected. However, as will be shown shortly, an affirmative answer, even in the case when $X^{*}$ has the Radon-Nikodým property, has a surprising consequence.

Anderson [1] asked whether the combination of rotundity and the RadonRiesz property implies midpoint local uniform rotundity. As noted above, he obtained a positive answer for reflexive spaces. Smith [19] answered this question negatively by giving a counterexample isomorphic to $l^{1}$. In spite of this example, it is still appropriate to ask for an affirmative response in a setting less restrictive than the reflexive case.

Question 11. If $X$ is a rotund Banach space with the Radon-Riesz property and whose dual space has the Radon-Nikodým property, then is $X$ midpoint locally uniformly rotund?

The next theorem establishes an affirmative response to this question for Lebesgue-Bochner function spaces. Note that, by a result of Sundaresan [23] (also see [7, VII. 2.9] and [24]), to assume the dual of $L^{p}(\mu, X)$ has the Radon-Nikodým property is no more than assuming its subspace $X^{*}$ has the same property. Moreover, the theorem gives an unqualified affirmative response to Question 11 if Question 10 has a positive answer whenever $X^{*}$ has the Radon-Nikodým property. Also note the theorem is a strengthening of Theorem 8 under the restriction on $X^{*}$.

THEOREM 12. Let $(S, \Sigma, \mu)$ be a finite measure space that is not purely atomic and let $1<p<\infty$. If $L^{p}(\mu, X)$ has the Radon-Riesz property and its dual space has the Radon-Nikodym property, then $X$ (and hence $L^{p}(\mu, X)$ ) is midpoint locally uniformly rotund.

The proof of the theorem is expedited by the following characterization of midpoint local uniform rotundity.

LEMMA 13. A Banach space $X$ is midpoint locally uniformly rotund if and only if the conditions $\|w\|=1,\left\|w+z_{n}\right\| \rightarrow 1$ and $\left\|w-z_{n}\right\| \rightarrow 1$ imply $z_{n} \rightarrow 0$.

Proof. The standard normalization argument shows that $X$ is midpoint locally uniformly rotund if and only if the conditions $\|w\|=1,\left\|x_{n}\right\| \rightarrow 1$, $\left\|y_{n}\right\| \rightarrow 1$, and $\left\|x_{n}+y_{n}-2 w\right\| \rightarrow 0$ imply $x_{n}-y_{n} \rightarrow 0$.

Now assume $X$ is midpoint locally uniformly rotund and suppose $\|w\|=1$ and $\left\|w \pm z_{n}\right\| \rightarrow 1$. Let $x_{n}=w+z_{n}$ and $y_{n}=w-z_{n}$. Then $\left\|x_{n}\right\| \rightarrow 1,\left\|y_{n}\right\|$ $\rightarrow 1$ and $\left\|x_{n}+y_{n}-2 w\right\|=0$ and hence $x_{n}-y_{n}=2 z_{n} \rightarrow 0$.

Conversely, suppose $\|w\|=\left\|x_{n}\right\|=\left\|y_{n}\right\|=1$ and $\left\|x_{n}+y_{n}-2 w\right\| \rightarrow 0$. 
Let $z_{n}=x_{n}-w$ and note $\left\|w+z_{n}\right\|=1$. Also $\left\|w-z_{n}\right\| \rightarrow 1$ since $\mid(1-\| w$ $\left.-z_{n} \|\right)|=|\left(\left\|y_{n}\right\|-\left\|2 w-x_{n}\right\|\right) \mid<\left\|x_{n}+y_{n}-2 w\right\|$. By the hypothesis it follows that $z_{n} \rightarrow 0$, that is, $x_{n} \rightarrow w$. Similarly, letting $z_{n}=y_{n}-w$, it follows that $y_{n} \rightarrow w$ and hence $x_{n}-y_{n} \rightarrow 0$.

Proof OF TheOREM 12. If $X$ is not midpoint locally uniformly rotund, then by Lemma 13 there exist $w$ and $\left(z_{n}\right)$ in $X$ and $c>0$ such that $\|w\|=1$, $\left\|w \pm z_{n}\right\| \rightarrow 1$ and $\left\|z_{n}\right\| \geqslant c$. Let $T \in \Sigma$ be a set of positive measure that contains no atoms and let $r_{n}$ denote the " $n$th Rademacher function" defined on $T ; r_{n}(s)=0$ for $s \in S \backslash T$. Again assume $\mu(S)=1$. Define $f_{n}$ and $f$ in $L^{p}(\mu, X)$ by $f_{n}(s)=w+r_{n}(s) z_{n}$ and $f(s)=w$ for $s \in S$. It is easy to verify that $\|f\|_{p}=1,\left\|f_{n}\right\|_{p} \rightarrow 1$ and $\left\|f_{n}-f\right\|_{p}>c \mu(T)^{1 / p}>0$. Since the dual of $L^{p}(\mu, X)$ is $L^{q}\left(\mu, X^{*}\right)$ where $p^{-1}+q^{-1}=1$ and since simple functions are dense in $L^{q}\left(\mu, X^{*}\right)$, to show $\left(f_{n}\right)$ converges weakly to $f$ it suffices to show $\left\langle x^{*} \chi_{E}, f_{n}-f\right\rangle \rightarrow 0$ for all $x^{*} \in X^{*}$ and $E \in \Sigma$. But this follows since $\left(z_{n}\right)$ is bounded in $X$ and $\left(r_{n}\right)$ converges weakly to zero in $L^{1}(\mu)$. Hence $L^{P}(\mu, X)$ does not have the Radon-Riesz property and the proof of Theorem 12 is complete.

The final generalizations of uniformly rotund spaces considered here are uniformly non- $l^{1}(n)$ and $B$-convex Banach spaces. The notion of $B$-convexity was introduced by Beck [2] in order to obtain a strong law of large numbers for certain vector-valued random variables. A Banach space $X$ is said to be uniformly non- $l^{1}(n)$ if there exists $\varepsilon>0$ such that for each $n$ elements $x_{1}, \ldots, x_{n}$ of the unit ball of $X,\left\|x_{1} \pm \cdots \pm x_{n}\right\|<n(1-\varepsilon)$ for some choice of signs. A Banach space is $B$-convex if it is uniformly non- $l^{1}(n)$ for some $n$. The main step of our argument showing that the notion uniform non- $l^{1}(n)$ lifts from $X$ to $L^{p}(\mu, X)$ for $1<p<\infty$ is contained in the following lemma. If $X$ is one-dimensional, the inequality follows from results of Sundaresan [21], [22].

LEMMA 14. If a Banach space $X$ is uniformly non- $l^{1}(n)$ and $1<p<\infty$, then there exists a constant $\alpha$ with $0<\alpha<1$ such that if $x_{1}, \ldots, x_{n}$ are elements of $X$, then

$$
\sum\left\|\frac{x_{1} \pm \cdots \pm x_{n}}{n}\right\|^{p}<\frac{2^{n-1} \alpha}{n}\left(\left\|x_{1}\right\|^{p}+\cdots+\left\|x_{n}\right\|^{p}\right)
$$

where the sum is taken over all $2^{n-1}$ choices of signs.

Proof. For economy of space, denote the sum on the left-hand side of the inequality by $\sigma$. Also assume, without loss of generality, that $\left\|x_{1}\right\|=1>\left\|x_{j}\right\|$ for $j=2, \ldots, n$. Let $\varepsilon>0$ be the $\varepsilon$ in the definition of $X$ being uniformly non- $l^{1}(n)$. Then one of the $2^{n-1}$ terms in $\sigma$ is less than $(1-\varepsilon)^{p}$. The triangle inequality and the convexity of the function $\Phi(t)=|t|^{p}$ on the other $2^{n-1}-1$ terms then yields the inequality

$$
\sigma<(1-\varepsilon)+\left(\left(2^{n-1}-1\right) / n\right)\left(\left\|x_{1}\right\|^{p}+\cdots+\left\|x_{n}\right\|^{p}\right) .
$$


Therefore

$$
\frac{\sigma}{\left\|x_{1}\right\|^{p}+\cdots+\left\|x_{n}\right\|^{p}}<\frac{1-\varepsilon}{1+\left\|x_{2}\right\|^{p}+\cdots+\left\|x_{n}\right\|^{p}}+\frac{2^{n-1}-1}{n} .
$$

First consider the case that $\left\|x_{2}\right\|^{p}+\cdots+\left\|x_{n}\right\|^{p}>(n-1)(1-\varepsilon)$. In this case,

$$
\frac{\sigma}{\left\|x_{1}\right\|^{p}+\cdots+\left\|x_{n}\right\|^{p}}<\frac{1-\varepsilon}{1+(n-1)(1-\varepsilon)}+\frac{2^{n-1}-1}{n}<\frac{2^{n-1}}{n} .
$$

Now consider the case that $\left\|x_{2}\right\|^{p}+\cdots+\left\|x_{n}\right\|^{p}<(n-1)(1-\varepsilon)$. The function $\Phi(t)=|t|^{p}$ is strictly convex. Thus, if $0<t_{2}, \ldots, t_{n}<1$ and at least one of the $t_{j}$ 's is strictly less than 1 , then

$$
\left(\frac{1+t_{2}+\cdots+t_{n}}{n}\right)^{p}<\frac{1}{n}\left(1+t_{2}^{p}+\cdots+t_{n}^{p}\right) .
$$

By the compactness of the set $\left\{\left(t_{2}, \ldots, t_{n}\right) \in[0,1]^{n-1}: t_{2}^{p}+\cdots+t_{n}^{p}<(n\right.$ $-1)(1-\varepsilon)\}$, there exists a constant $\beta$ with $0<\beta<1$ such that

$$
\left(\frac{1+t_{2}+\cdots+t_{n}}{n}\right)^{p}<\frac{\beta}{n}\left(1+t_{2}^{p}+\cdots+t_{n}^{p}\right)
$$

for each $\left(t_{2}, \ldots, t_{n}\right)$ in the above set. Therefore an application of the triangle inequality and the last inequality to the definition of $\sigma$ yields

$$
\begin{aligned}
\sigma & <2^{n-1}\left(\frac{1+\left\|x_{2}\right\|+\cdots+\left\|x_{n}\right\|}{n}\right)^{p} \\
& <\frac{2^{n-1} \beta}{n}\left(1+\left\|x_{2}\right\|^{p}+\cdots+\left\|x_{n}\right\|^{p}\right) .
\end{aligned}
$$

Setting $\alpha=\max \left\{\beta,\left(n / 2^{n-1}\right)\left((1-\varepsilon) /(1+(n-1)(1-\varepsilon))+\left(2^{n-1}-\right.\right.\right.$ $1) / n)\}$ completes the proof of Lemma 14.

In particular, note the theorem shows (when $n=2$ ) that the property uniformly nonsquare introduced by James [10] lifts from $X$ to $L^{p}(\mu, X)$ and $l^{p}(X)$ for $1<p<\infty$.

THEOREM 15. Let $(S, \Sigma, \mu)$ be a measure space. If $1<p<\infty$ and $X$ is a Banach space that is uniformly non- $l^{1}(n)$, then $L^{p}(\mu, X)$ is uniformly non- $l^{1}(n)$.

Proof. Suppose $\varepsilon>0$ is given in the definition of $X$ being uniformly non- $l^{1}(n)$. Let $f_{1}, \ldots, f_{n}$ be functions in $L^{P}(\mu, X)$ with $\left\|f_{j}\right\|<1$ for $j=$ $1, \ldots, n$. Then for $s \in S$

$$
\sum\left\|\frac{f_{1}(s) \pm \cdots \pm f_{n}(s)}{n}\right\|_{x}^{p}<\frac{2^{n-1} \alpha}{n}\left(\left\|f_{1}(s)\right\|^{p}+\cdots+\left\|f_{n}(s)\right\|^{p}\right)
$$

where $\alpha$ is given by Lemma 14. Integrating over $S$ yields $\Sigma \|\left(f_{1}\right.$ $\left.\pm \cdots \pm f_{n}\right) / n \|_{p}^{p}<2^{n-1} \alpha$. Since the sum contains $2^{n-1}$ terms, at least one of the terms is less than or equal to $\alpha$. Therefore $L^{p}(\mu, X)$ is uniformly 
non- $l^{1}(n)$ and the proof of Theorem 15 is complete.

Using a result of Giesy [9] which states that $X$ is not $B$-convex if and only if $l^{1}$ is finitely representable in $X$, Figiel [17] proved the following corollary. The proof of this result is immediate from Theorem 15.

Corollary 16 (Figiel). Let $(S, \Sigma, \mu)$ be a measure space. If $1<p<\infty$ and $X$ is a Banach space that is $B$-convex, then $L^{P}(\mu, X)$ is $B$-convex.

\section{REFERENCES}

1. K. W. Anderson, Midpoint local uniform convexity, and other geometric properties of Banach spaces, Dissertation, University of Illinois, 1960.

2. A. Beck, A convexity condition in Banach spaces and the strong law of large numbers, Proc. Amer. Math. Soc. 13 (1962), 329-334.

3. S. Bochner and A. E. Taylor, Linear functionals on certain spaces of abstractly-valued functions, Ann. of Math. (2) 39 (1938), 913-944.

4. M. M. Day, Some more uniformby convex spaces, Bull. Amer. Math. Soc. 47 (1941), 504-507.

5. Strict convexity and smoothness of normed spaces, Trans. Amer. Math. Soc. 78 (1955), 516-528.

6. __ Normed linear spaces, 3rd ed., Ergebnisse der Mathematik und ihrer Grenzgebiete, Band 21, Springer-Verlag, New York, 1973.

7. J. Diestel and J. J. Uhl, Jr., Vector measures, Math. Surveys, No. 15, Amer. Math. Soc., Providence, R. I., 1977.

8. A. L. Garkavi, The best possible net and best possible cross section of a set in a normed space, Izv. Akad. Nauk SSSR Ser. Mat. 26 (1962), 87-106 = Amer. Math. Soc. Transl. (2) 39 (1964), 111-132.

9. D. P. Giesy, On a convexity condition in normed linear spaces, Trans. Amer. Math. Soc. 125 (1966), 114-146.

10. R. C. James, Uniformly non-square Banach spaces, Ann. of Math. (2) 80 (1964), 542-550.

11. E. Leonard, Banach sequence spaces, J. Math. Anal. Appl. 54 (1976), 245-265.

12. E. Leonard and K. Sundaresan, Geometry of Lebesgue-Bochner function spaces-smoothness, Bull. Amer. Math. Soc. 79 (1973), 546-549.

13. A. R. Lovaglia, Locally uniformly convex Banach spaces, Trans. Amer. Math. Soc. 78 (1955), 225-238.

14. E. J. McShane, Linear functionals on certain Banach spaces, Proc. Amer. Math. Soc. 1 (1950), 402-408.

15. J. Radon, Theorie und Anwendungen der absolut additiven Mengen funktionen, Sitzungsber. Akad. Wiss. Wien 122 (1913), 1295-1438.

16. F. Riesz, Sur la convergence en moyenne. I, II, Acta Sci. Math. (Szeged) 4 (1928-1929), 58-64, 182-185.

17. H. P. Rosenthal, Some applications of p-summing operators to Banach space theory, Studia Math. 58 (1977), 21-43.

18. M. A. Smith, Products of Banach spaces that are uniformly rotund in every direction, Pacific J. Math. 73 (1977), 215-219.

19. Some examples concerning rotundity in Banach spaces, Math. Ann. 233 (1978), 155-161.

20. V. L. Śmul'yan, Sur la dérivabilité de la norme dans l'espace de Banach, Dokl. Akad. Nauk URSS 27 (1940), 643-648.

21. K. Sundaresan, Uniformly non- $l_{n}^{(1)}$ Orlicz spaces, Israel J. Math. 3 (1965), 139-146.

22. , Uniformly non-square Orlicz spaces, Nieuw Arch. Wisk. 14 (1966), 31-39.

23. The Radon-Nikody'm theorem for Lebesgue-Bochner function spaces, J. Functional Analysis 24 (1977), 276-279.

24. B. Turett and J. J. Uhl, Jr., $L_{p}(\mu, X)(1<p<\infty)$ has the Radon-Nikody'm property if $X$ does by martingales, Proc. Amer. Math. Soc. 61 (1976), 347-350. 
25. V. Zizler, On some rotundity and smoothness properties of Banach spaces, Dissertationes Math. (Rozprawy Mat.) 87 (1971).

Department of Mathimatics anid Statistics, Miami University, Oxford, Ohio 45056 (Current address of M. A. Smith)

Department of Mathematics, Texas Thi University, Lubbock, Texas 79409

Current address (Barry Turett): Department of Mathematical Sciences, Oakland University, Rochester, Michigan 48063 